\title{
Pertinence des sciences humaines et sociales en médecine
}

\author{
Relevance of medical humanities in health sciences curricula
}

La recherche de Maxence Gaillard et Nicolas Lechopier, menée sous la forme d'une enquête effectuée auprès de dix universités françaises, qui est publiée dans ce numéro ${ }^{[1]}$, révèle une disparité de la conception et de l'enseignement des sciences humaines en première année de médecine. Cet enseignement est obligatoire au même titre que les disciplines biomédicales; les disciplines des sciences humaines et sociales (SHS) participent donc à la sélection des étudiants en fin de première année, et déterminent en partie leur future trajectoire professionnelle. Les auteurs montrent que chaque faculté s'accommode à sa manière de l'impératif des SHS en fonction des moyens à disposition. Cet article propose une vision précise et élaborée, notamment pour le lecteur venu d'ailleurs, des contraintes et des réalités des universités françaises. Bien que cet article soit centré, et à juste titre, sur le modèle hexagonal, il met en évidence, implicitement ou explicitement, des problèmes qui sont posés de manière plus générale à tous les responsables d'un enseignement de sciences humaines en médecine. Ces problèmes sont liés à des aspects pragmatiques, comme la taille d'une faculté de médecine, comme les forces disponibles pour délivrer ces cours, ou encore comme les modalités d'évaluation plus ou moins conformes avec les exigences d'un travail rédactionnel.

Mais cet article passe comme chat sur braise quand il s'agit de donner une définition précise des sciences humaines en médecine, comme si la chose était acquise. De même, il ne discute pas la question des objectifs réalistes d'un tel enseignement. En effet, ceux qui sont posés par le texte officiel de l'unité d'enseignement UE7 sont tellement démesurés qu'ils forcent à opérer des choix. Par ailleurs le consensus autour d'objectifs aussi vagues que creux (développer la réflexion des étudiants ou encore encourager leur humanité) est si peu satisfaisant, que soit on est dans l'évidence, concernant la réflexion, soit on est dans la non pertinence : l'aspect humain d'un individu n'est pas automatiquement lié à un savoir issu des sciences humaines et sociales.

On pourrait penser que je souligne ici une faiblesse de cet article, ce qui n'est pas le cas, au contraire. L'approche pragmatique choisie par les auteurs prévient un enlisement conceptuel et évite les inévitables et lassantes querelles autour de la nécessité d'une discipline ou d'une autre, querelles qui relèvent plus d'un instinct territorial et budgétaire que d'une pertinence intellectuelle. Les tentatives de définition des SHS mènent le plus souvent à des confrontations stériles entre experts, et nous décentrent du plus important, soit le travail de terrain. Ce dernier exige l'élaboration d'un enseignement qui soit en adéquation avec la faculté de médecine dans laquelle il s'inscrit, avec la formation des enseignants, et avec le format pédagogique et le nombre d'heures qui sont mises à disposition tout au long des études. Comme le relèvent les auteurs, il n'y a pas de contenu ni de format adéquat pour toutes les écoles de médecine, c'est à chacun nous de créer le modèle le plus pertinent. 
En revanche, il me semble que nous pourrions mieux nous concerter pour définir des objectifs spécifiques que devraient poursuivre les SHS, quels que soient le pays et la faculté dans lesquels elles s'inscrivent. C'est du moins ce que nous avons tenté de faire, il y a quelques années, durant trois jours d'atelier ${ }^{1}$. Une quinzaine de personnes concernées (venues d'Europe et des États-Unis), des cliniciens, des enseignants issus de plusieurs disciplines des sciences humaines, des vice-doyens responsables de l'enseignement se sont réunies à cet effet. Les discussions ont été parfois tumultueuses mais toujours constructives. Nous avons convenu qu'il était important de : a) nourrir et renforcer les aptitudes interprétatives et réflexives des étudiants ; b) affiner le jugement des étudiants pour mieux leur permettre d'appréhender la singularité de la relation clinique ; c) être capable de construire un argumentaire pour que chaque médecin puisse justifier de ses actes envers lui-même et envers les autres.

Des objectifs communs, discutés puis validés par tous, contribuent à renforcer notre présence et à donner un sens spécifique à notre enseignement dans une faculté de médecine. Interpréter, argumenter, mettre à distance, formuler avec précision sont des aptitudes intellectuelles très développées dans le cadre d'une recherche en science humaine ou sociale, quelle qu'elle soit. La plupart de nos compétences s'inscrivent dans un registre intellectuel plus que moral, mais les deux sont interdépendants ; par exemple, une attention précise portée au langage des patients (et pas seulement à son récit), sous-tendue par une rigueur quasi littéraire, permet d'intégrer l'aspect singulier de son expérience par une analyse précise de son discours. C'est dans cette précision de l'écoute, et du jugement formé à partir de cette écoute, que la réponse du médecin sera plus adéquate, autant sur le plan thérapeutique que sur le plan relationnel.

À propos d'objectif toujours, les SHS jouent un rôle important dans le maintien de la curiosité des étudiants en médecine. Ils sont nombreux à sortir d'une

1. Dans le cadre du World Knowledge Dialogue : Interdisciplinarity in Action. A Practical Experience of Interdisciplinarity Research. Villars-sur-Ollon, 10-14 octobre 2010. formation générale (baccalauréat) avec une curiosité pour différents domaines et notre enseignement participe à maintenir éveillés ces différents intérêts. Comme l'écrivait Montaigne: «La plupart des esprits ont besoin de matières étrangères pour se dégourdir et exercer». " Dégourdir et exercer» l'esprit des étudiants c'est participer à leur agilité intellectuelle, à la finesse et la subtilité de leur jugement, probablement aussi à leur créativité qui peut se montrer utile dans les situations cliniques complexes.

Le deuxième article discuté ici, écrit par Gaëtan Absil et Patrick Govers ${ }^{[2]}$, pose la question de l'enseignement de l'histoire de la médecine destiné aux étudiants en médecine, et remet en cause celle qui est écrite par des médecins, notamment dans la rédaction des manuels. Le débat est ancien et figé ; les historiens se plaignent des médecins qui s'improvisent historiens du dimanche, les médecins se plaignent des historiens qui sont peut-être experts mais ont de la difficulté à atteindre un public de jeunes étudiants en médecine.

Pour sortir de ces vieux clivages, on pourrait poser le problème autrement et réfléchir à la question de la pertinence de l'histoire de la médecine (question qui devrait se poser pour toutes les disciplines appartenant aux SHS). Se résume-t-elle à transmettre un contenu, un socle de connaissances que tous les étudiants devraient avoir intégrées ? Auquel cas il faudrait le définir. Ou l'histoire constitue-t-elle un outil intellectuel appartenant à l'archipel des SHS, qui de ce fait contribuerait à remplir les objectifs dont il est question plus haut? Si à mes yeux c'est cette dernière question qui doit retenir toute notre attention, celle du contenu ne peut pas être évacuée entièrement. En effet, il est important qu'enseignants et étudiants puissent s'appuyer sur un ou des manuels qui remplissent les critères de la recherche académique et qui, directement ou indirectement, touchent à des préoccupations contemporaines. A cet égard, le manuel Médecine, Santé et Sciences humaines ${ }^{[3]}$, publié sous la direction du Collège des enseignants de sciences humaines et sociales en médecine et en santé, est exemplaire car il propose un contenu thématique d'une grande richesse, rédigé par un ensemble d'auteurs compétents dans leur domaine, quelle que soit leur formation. S'il est vrai que la plupart du 
temps les médecins n'ont pas l'outillage méthodologique et historiographique suffisant pour écrire l'histoire de manière professionnelle, certains font exceptions et écrivent des ouvrages qui font référence (comment ne pas penser à Owsei Temkin à Jean Starobinski ?). Là encore, les querelles de clochers prennent un temps précieux qu'il est préférable de consacrer à l'examen des contenus.

Mais les deux auteurs ne se laissent pas enfermer dans ce débat et proposent aussi plusieurs règles intéressantes pour actualiser les pratiques de l'enseignement de l'histoire dans les sciences de la santé. La question de l'historicité (règle C), qui vise à une mise à distance du milieu culturel dans lequel nous évoluons pour considérer d'autres systèmes de valeurs, d'autres philosophies du corps, de la santé et de la maladie, est fondamentale. Elle nous permet de réaliser que notre compréhension du monde est largement déterminée par le cadre culturel auquel on appartient ; elle agit comme un produit de contraste aidant les étudiants à mieux prendre conscience du cadre dans lequel ils vont pratiquer. Souligner l'influence de la culture sur l'exercice de la médecine n'implique pas scepticisme et relativisme idéologique. L'analyse d'une consultation au $18^{\mathrm{e}}$ siècle, par exemple, révèle aux étudiants par contraste les éléments et les déterminants de la consultation contemporaine, et leur permet d'en prendre la juste mesure.

La pluridisciplinarité (règle E) est elle aussi cruciale, mais combien difficile à appliquer si on veut éviter une simple apposition de points de vue ou une histoire qui répondrait uniquement à des questions contemporaines. Elle est d'autant plus difficile quand elle associe la culture médicale et la culture des lettres et des SHS. Les parcours intellectuels et académiques sont si différents les uns des autres, de même que les finalités facultaires, notamment en termes d'applicabilité des savoirs, qu' une familiarisation et une reconnaissance de la légitimité des deux formats s'impose avant de pouvoir créer un dialogue constructif. Le team-teaching (un clinicien et un chercheur en SHS) offre une solution satisfaisante pour combler cette difficulté. Il implique une préparation à deux, une négociation (voire une confrontation dans le sens le plus noble du terme) à refaire pour chaque étape : choix du thème, du matériel, du contenu, des messages. Ce sont ces étapes concrètes qui favorisent la compréhension et l'estime mutuelle, qui garantissent un enseignement dialogique qu'aucune des deux parties n'auraient pu produire à elle-seule. En outre, elles nous protègent d'un amalgame superficiel de connaissances que ne valideraient aucune des deux cultures.

Ces deux articles, au-delà de leur intérêt spécifique, soulèvent des questions qui nous concernent tous et qui exigent des réponses nuancées en fonction du contexte pédagogique dans lequel chacun d'entre nous inscrit un programme de SHS. Depuis près de deux décennies, les sciences humaines et sociales cherchent (et souvent parviennent) à s'implanter dans les Ecoles de médecine européennes. Cela semble momentanément acquis; cet oxymore indique une reconnaissance et une satisfaction, mais aussi une vulnérabilité.

Pour les consolider, il me semble que nous devons nous détourner du combat lié à la notion vague et macroscopique de leur «utilité », et argumenter leur essor en fonction de leur pertinence. En effet, comment définir ce terme d'utilité ? Utile en fonction ou en comparaison de quoi ? Et pour qui ? La culture, au sens large du terme, doit-elle être directement et immédiatement utile dans une collectivité ? Le terme d'utilité renvoie à un débat idéologique basé sur des valeurs abstraites et mouvantes. En pédagogie, il tourne parfois au diktat (à quoi ça sert la littérature ?) ce qui empêche les SHS de donner le meilleur d'elles-mêmes et d'apporter une complémentarité intellectuelle à la biomédecine. Pour autant, elles ne peuvent pas non plus se réduire à un alibi, ni à un vernis culturel, ni à un divertissement sympathique pour étudiants surmenés, ni à une pseudo contre-culture. Les écueils sont nombreux.

Si l'utilité n'est pas un concept satisfaisant, il peut être remplacé avantageusement par celui de pertinence. La question n'est plus alors de savoir si les SHS sont utiles, mais comment les rendre pertinentes pour la formation des futurs médecins. S'il est aisé de trouver des thèmes intéressants pour l'enseignement des SHS, il est nettement plus difficile d'expliciter aux étudiants (et aux autorités pédagogiques) leur pertinence. La pertinence renvoie à l'adaptation de ces thèmes au public visé, elle dépend de l'organisa- 
tion pédagogique de la faculté, elle nécessite d'établir un lien conceptuel explicite entre une thématique et la pratique future des étudiants. Elle permet ainsi la multiplicité et la diversité des programmes tout en maintenant une cohésion entre ces programmes par l'adoption d'objectifs spécifiques communs.

\section{Micheline LOUIS-COURVOISIER}

Historienne, Institut Ethique-Histoire-Humanités Responsable du programme de sciences humaines en médecine

Faculté de médecine, Université de Genève (Centre médical universitaire, 1211 Genève 4 , Suisse) Mailto : Micheline.Louis-Courvoisier@unige.ch

\section{Références}

1. Gaillard M, Lechopier N. Relever le défi d'introduire aux sciences humaines et sociales en première année commune des études de santé. Mise en perspective de quelques pratiques pédagogiques. Pédagogie Médicale 2015;16:23-34

2. Absil G, Govers P. Comment écrire l'histoire de la médecine pour les étudiants des sciences de la santé ? Pédagogie Médicale 2015;16:9-22

3. Collège des enseignants de sciences humaines et sociales en médecine et en santé (Sous la direction de). Médecine, Santé et Sciences humaines. Paris : Belles-Lettres, 2011. 